\title{
Déprovincialiser les universités européennes
}

Deprovincializing European universities

Desprovincializar las universidades europeas

\section{Caroline Rolland-Diamond, Capucine Boidin, James Cohen et Ramon Grosfoguel}

\section{(2) OpenEdition}

\section{Journals}

Édition électronique

URL : https://journals.openedition.org/ideas/278

DOI : 10.4000/ideas. 278

ISSN : 1950-5701

Traduction(s) :

Deprovincializing European universities - URL : https://journals.openedition.org/ideas/2292 [en] Desprovincializar as universidades europeias - URL : https://journals.openedition.org/ideas/3048 [pt] Desprovincializar las universidades europeas - URL : https://journals.openedition.org/ideas/3053 [es]

Éditeur

Institut des Amériques

Référence électronique

Caroline Rolland-Diamond, Capucine Boidin, James Cohen et Ramon Grosfoguel, « Déprovincialiser les universités européennes », IdeAs [En ligne], 2 | 2012, mis en ligne le 25 juin 2012, consulté le 19 octobre 2022. URL : http://journals.openedition.org/ideas/278 ; DOI : https://doi.org/10.4000/ideas 278

Ce document a été généré automatiquement le 19 octobre 2022.

Creative Commons - Attribution - Pas d'Utilisation Commerciale - Pas de Modification 4.0 International - CC BY-NC-ND 4.0

https://creativecommons.org/licenses/by-nc-nd/4.0/ 


\title{
Déprovincialiser les universités européennes
}

\author{
Deprovincializing European universities \\ Desprovincializar las universidades europeas
} Caroline Rolland-Diamond, Capucine Boidin, James Cohen et Ramon
Grosfoguel

\section{Les universités européennes à la croisée des chemins}

1 Le classement de Shanghai publié en 2003 a touché la fierté française en plein cœur en reléguant ses universités et grandes écoles en bas du tableau d'honneur, loin derrière les universités des mégalopoles nord-américaines, britanniques et japonaises. Mille critiques furent formulées à l'encontre de ce classement. La plus importante est probablement l'avantage comparatif dont jouissent les publications en langue anglaise dans la compétition internationale et la difficulté à hiérarchiser les universités dans le domaine des sciences humaines et sociales. Pourtant l'analyse actualisée de plusieurs classements mondiaux, effectuée par Hervé Théry pour notre rubrique éclairage, invite d'abord à décentrer notre regard: "Pour les quatre classements retenus, la concentration des universités classées dans quelques régions du monde est telle qu'il a fallu ajouter au planisphère trois zooms détaillant la situation des États-Unis, de l'Europe et du Japon. » Ce dossier convie les lecteurs francophones à élargir leur champ de préoccupations non pas au seul «déclin» ou à l'«in-comparabilité » du modèle français, mais aussi aux inégalités mondiales ${ }^{1}$, en particulier au déséquilibre entre les universités nord- et sud-américaines, même si des universités brésilienne, mexicaine, argentine et chilienne apparaissent dans certains classements ${ }^{2}$.

Des classements, il faudrait en faire usage comme du sel, évitant l'excès de zèle ou le rejet stérile, pour reprendre l'expression de Boaventura de Sousa, dont les lecteurs trouveront ici les réflexions et les propositions fortes pour les universités européennes dans la section Eclairages de ce numéro. Penser la situation des universités européennes, à l'aune des expériences américaines, est l'objectif général de ce dossier. 
L'influence du modèle américain d'enseignement supérieur s'est d'abord faite sentir en Amérique latine avant de toucher l'Europe. La libéralisation du marché de l'enseignement supérieur, la multiplication des agences d'évaluation mais aussi la création d'institutions universitaires spécialisées pour certaines minorités sont mises en place en Amérique latine dès les années 1990. L'article de Manuela Boatca dans ce dossier montre justement la manière dont les "initiatives d'excellence" en Allemagne sont pensées comme un moyen pour rattraper le nouvel occident, le modèle étatsunien d'enseignement supérieur. La sensation d'avoir à "rattraper" un retard est ainsi forte au sein du système universitaire européen, alors même que l'impulsion de depart pour la création de cet espace commun d'enseignement supérieur avait d'autres racines.

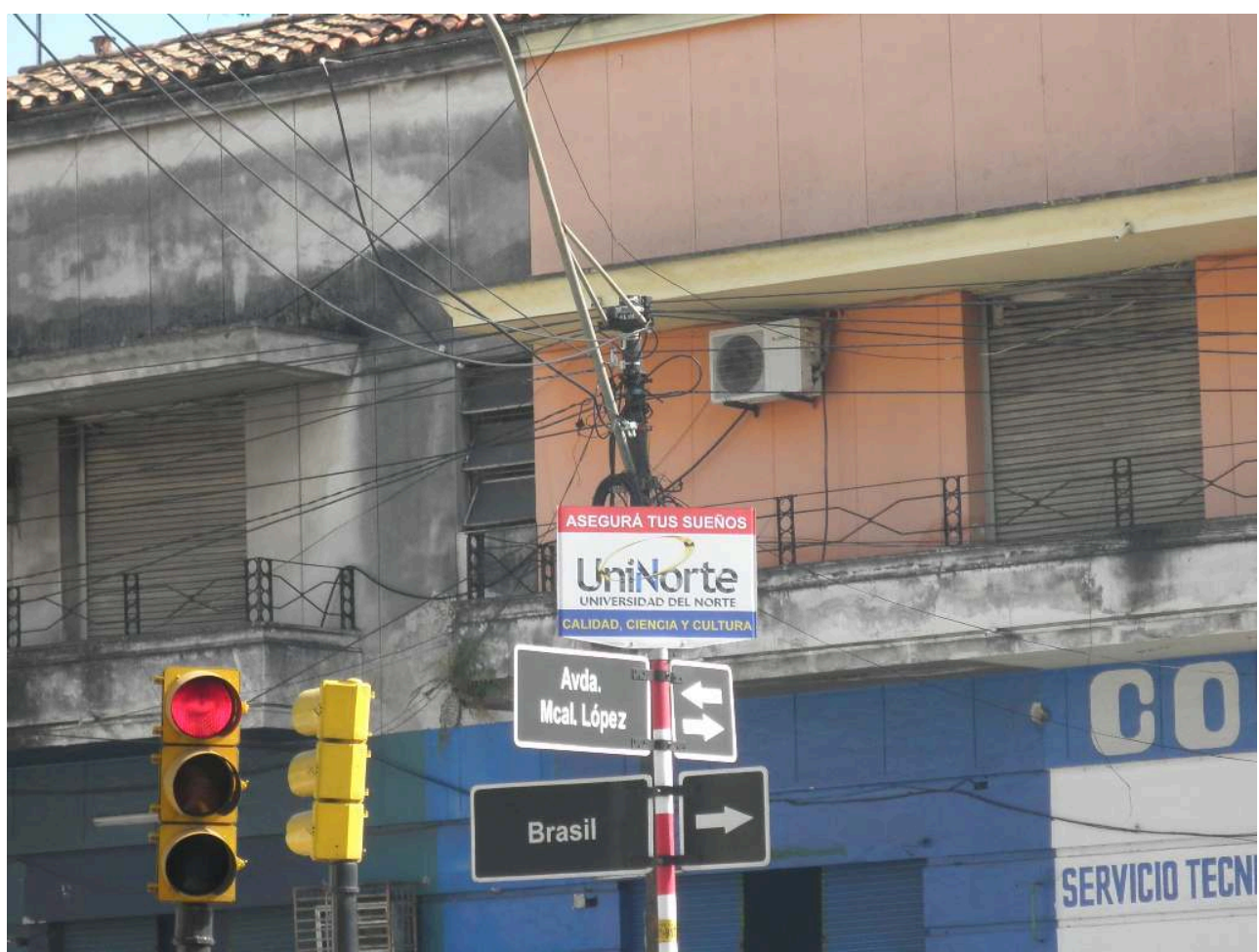

Une publicité omniprésente dans la ville d'Asunción pour UNINORTE, une université privée : «Assuretoi de réaliser tes rêves », Université du Nord, Qualité, Science et Culture. L'impératif est accentué selon la prononciation paraguayenne, influencée par la langue guarani : « Asegurá ».

Photo : C. Boidin, Paraguay, 2011

3 Pour mémoire, le projet de créer un espace européen de l'enseignement supérieur a été lancé en Sorbonne en $1998^{3}$ : «l'Europe que nous bâtissons n'est pas seulement celle de l'Euro, des banques et de l'économie ; elle doit être aussi une Europe du savoir.» Ce processus a été officiellement lancé en 1999 à Bologne, avec la signature de 29 pays. Mais s'ajoute alors dans la déclaration de Bologne un objectif absent en 1998: " la coopération européenne dans l'assurance qualité ». Que signifient ces deux mots assemblés, "assurance et qualité »? "Dans l'enseignement supérieur, l'assurance qualité renvoie aux politiques, procédures et pratiques conçues en vue de la garantie, du maintien ou de l'amélioration de la qualité telle qu'elle s'entend dans un contexte spécifique». ${ }^{4}$ Dans ce dispositif, l'autonomie et la responsabilité financière des établissements comme le caractère externe de l'évaluation sont les deux piliers permettant de "rassurer" les différents partenaires quant à la qualité de leurs établissements universitaires. En 2007, la création du Registre européen pour la 
garantie de la qualité (EQAR) a précipité la création de structures nationales d'évaluation de la qualité dans les universités : en France, par exemple, l'AERES (Agence d'Evaluation de l'Enseignement et de la Recherche).

Les universités ont alors été conçues comme étant gouvernables à la manière des entreprises : par la méthode du benchmarking (la mise au point d'objectifs chiffrés à atteindre - en urgence), décryptée par Isabelle $\mathrm{Bruno}^{5}$, les individus et les organisations sont sommés de se soumettre à ces objectifs et mis en concurrence les uns avec les autres. Le financement « d'initiatives d'excellence», ou pour le dire plus clairement le financement "ciblé » d'institutions et de programmes, rentre dans cette logique de mise en compétition. La coopération peut devenir complexe à mettre en œuvre et toute réflexion de fond impossible à mener : la course aux financements, aux objectifs et aux remises de rapports monopolisent toutes les énergies et remplissent tous les agendas des institutions d'enseignement supérieur. Comme le résume Boaventura de Sousa :

«In a few decades the university went from producing knowledge and professionals for the market, to becoming itself a market, the market of tertiary education, and finally, at least according to powerful visionaries, to being run like a market organization, a business organization. "

Le deuxième et dernier grand objectif ajouté au projet initial défini en Sorbonne - celui de la « dimension sociale de l'enseignement supérieur » - n'est clairement défini qu'en 2007 par le Communiqué de Londres qui déclare, enfin, que «le corps étudiant qui accède à l'enseignement supérieur, y fait ses études et les achève, à tous les niveaux devrait refléter la diversité de nos populations $»^{6}$. Or dans ce domaine, en 2010, le bilan est plus mitigé que pour la création d'un cadre commun de référence ${ }^{7}$ ou la mise en place de l'assurance qualité, eu égard aux critères de bonne gouvernance (objectifs chiffrés et donc mesurables) :

«Très peu de pays ont fixé des objectifs chiffrés spécifiques pour améliorer la participation des groupes sous-représentés dans l'enseignement supérieur et la moitié seulement des pays de Bologne contrôlent de façon systématique leur participation. Les mesures nationales les plus fréquentes pour élargir la participation consistent à apporter un soutien financier ciblé et à créer des filières d'accès et/ou des procédures d'admission parallèles. (...) Dans les pays signataires du processus de Bologne, la sous-représentation est le plus souvent liée à l'origine socio-économique ou au niveau d'études des parents, au statut de minorité ou au handicap. (...) Les différentes approches adoptées pour identifier les groupes sousreprésentés montrent qu'il peut s'agir d'un domaine très sensible, rendant impossible toute comparaison paneuropéenne dans la pratique. » (nous soulignons)

Autrement dit, sont mis en place des financements ciblés sur certains membres de catégories définies pour leur permettre d'entrer dans certaines institutions d'élites susceptibles d'être compétitives au niveau international - diminuant par là même les investissements dans le reste des institutions et renforçant les processus de marginalisation, censés être corrigés par cette politique. La panacée serait une université d'élites "diversifiées", grâce à des filières d'accès parallèles. L'objectif affiché est de refléter la diversité de la population. Pas de promouvoir l'égalité des chances pour tous.

7 Finalement, depuis 1999, de nouveaux processus de rationalisation sont impulsés au cœur des universités européennes: multiplication de critères chiffrables et «évaluables » pour mesurer, classer et financer l'activité des universités, que ce soit du côté des enseignements ou des publications; recherche de la "diversité ", comme critère de justice sociale. Deux dispositifs, de nature différente, qui sont propres au 
fonctionnement du modèle nord-américain d'enseignement supérieur, mais qui au fond résultent d'une histoire particulière.

\section{Au-delà du modèle ou du repoussoir. le système d'enseignement supérieur états-unien en perspective}

8 Modèle ou repoussoir, le système d'enseignement supérieur états-unien est pourtant souvent mal connu. Un rappel de ses caractéristiques principales en ouverture de ce dossier sera ainsi utile. Formés pendant la période de l'Amérique coloniale, les premiers colleges qui deviendront ensuite, du fait de leur ancienneté vénérable, les universités dites de l'Ivy League, sont des établissements privés. Leur fonction consiste à l'origine à former les élites politiques et spirituelles des colonies britanniques. La division territoriale en treize colonies relativement autonomes explique la multiplication de ces institutions qui, sur le modèle d'Oxford et Cambridge, développent un système de campus résidentiel pour leurs étudiants. L'indépendance acquise, la jeune République reconnaît immédiatement (Ordonnance du Nord-Ouest de 1785) la nécessité sociale de se doter d'institutions publiques d'enseignement dans chacun des nouveaux Etats créés. S'il s'agit avant tout à cette époque de développer un système d'écoles publiques élémentaires, le double principe de la responsabilité sociale des Etats en matière de scolarité publique et de son utilité pour la société est d'emblée posé avant de s'étendre à l'ensemble du système d'enseignement. Très vite, un système multiple se met en place mêlant des établissements entièrement privés et d'autres financés par l'Etat fédéré où ils se situent. Caractéristique importante, en effet, aux Etats-Unis, l'éducation relève des pouvoirs des Etats fédérés, pas de l'Etat central.

9 La période allant de 1785 à 1860 se caractérise par une innovation extrême et un fort consumérisme, sans quasiment la moindre supervision ou réglementation de l'Etat fédéral. Ce n'est pourtant pas une période de chaos mais plutôt de multiplication des établissements et d'innovation disciplinaire. On passe ainsi, à l'échelle du pays, de 25 établissements conférant un diplôme supérieur à 241 en 1860 . Outre la création de nouveaux colleges, la période est aussi celle où voient le jour d'autres types d'institutions: les universités proprement dites, les académies, les séminaires, les écoles scientifiques, les écoles normales et les instituts. Parallèlement à ce foisonnement, le programme enseigné dans les colleges est de temps à autre étendu audelà du « liberal arts» (mélange de latin, grec, mathématiques, auxquels s'ajoutent la science, la philosophie, l'histoire, ainsi que la composition et la littérature anglaises) pour inclure la médecine, le droit, l'ingénierie, la science militaire, le commerce, la théologie et l'agriculture.

\section{Naissance des universités publiques et des universités réservées aux minorités ( $\mathrm{mi}$ XIXe)}

Dans la deuxième moitié du XIX ${ }^{e}$ siècle, on assiste à un double phénomène : d'une part le renforcement du maillage d'établissements d'enseignement supérieur (avec la naissance de nouvelles universités publiques et des colleges et universités urbains) et d'autre part, la création d'institutions spécialement destinées à permettre l'accès à l'enseignement supérieur de groupes exclus jusqu'alors: les femmes ou les noirs par exemple. 
11 Tout d'abord, la loi Morrill de 1862 marque un tournant : elle officialise la création de ce qu'on appelle les « land-grant colleges ", autrement dit la création d'établissements d'enseignement supérieur sur des terres concédées à cette fin par l'Etat fédéré. Grâce à cette loi, les principales grandes universités publiques du pays voient le jour. C'est l'une des grandes étapes de la démocratisation de l'enseignement supérieur, désormais facile d'accès (tant du fait du coût peu élevé des études que de la proximité géographique) à un grand nombre de jeunes gens et de jeunes filles.

12 Tandis que les land-grant colleges et les universités d'Etat permettent aux jeunes de nombreuses régions rurales et petites villes d'accéder enfin à l'enseignement supérieur grâce à leur proximité géographique et leur coût raisonnable, dans les grandes villes, un mouvement similaire apparaît avec la création de colleges et universités municipaux. La tendance est en effet à bâtir également des établissements publics gratuits ou quasigratuits dans les centres urbains ${ }^{8}$. L'objectif est de répondre aux besoins des étudiants des villes en offrant notamment des cours innovants en formation technique industrielle avancée ou en commerce, en plus des cours classiques de «liberal arts». Ce type d'établissement permet pour la première fois aux étudiants des centres urbains n'ayant pas les moyens de s'inscrire à temps plein dans un college résidentiel (avec campus) de suivre des études tout en continuant d'habiter chez leurs parents et/ou de travailler grâce au développement des cours du soir et aux dispositifs pour les étudiants à temps partiel. Peu onéreux, ces colleges urbains de proximité se vont vite taillé une place unique et indispensable dans le paysage de l'enseignement supérieur américain.

13 L'accès des femmes à l'enseignement supérieur s'accélère après la Guerre de Sécession alors qu'il était limité auparavant à de rares "séminaires » ou « académies » ouverts aux femmes. Le processus de démocratisation prend deux formes: la création d'institutions spécifiques, les coordinate colleges, séparés mais liés à des colleges masculins (ainsi Radcliffe est lié à Harvard, Barnard à Columbia, ou encore Newcomb à Tulane en Louisiane). Parallèlement, un mouvement de développement de la mixité se met en place ${ }^{9}$. Fin XIX ${ }^{\mathrm{e}}$, près des trois quarts des colleges et universités du pays sont ouverts aux femmes, même si le nombre d'étudiantes ne représente encore que $2,5 \%$ de l'ensemble des jeunes femmes américaines.

14 La lutte des femmes pour accéder à l'enseignement supérieur coïncide avec le début du mouvement d'extension des opportunités éducatives aux noirs. Avant la Guerre de Sécession, seuls 29 étudiants noirs ont pu obtenir un diplôme d'enseignement supérieur. Après la guerre, la plupart des initiatives sont initialement d'ordre caritatif ou religieux. Vers la fin du siècle, les fondations philanthropiques de grandes entreprises et des bienfaiteurs privés fortunés apportent leur soutien au développement des colleges noirs. Au total, sans doute 200 établissements privés ou religieux destinés aux étudiants noirs sont créés dans les années 1870 et 1880 mais la très grande majorité n'est en réalité rien d'autre que des établissements dispensant un enseignement secondaire, palliant le manque de lycées pour Afro-Américains, et ils disparaissent vite faute de financements suffisants. En filigrane se pose ici un problème essentiel pour l'accès des noirs à l'enseignement supérieur dans l'Amérique de la ségrégation : la nature de l'enseignement dispensé et son adaptation au niveau d'études réel des jeunes noirs à la fin du XIXe siècle.

15 L'intention originelle des fondateurs des colleges noirs est de fournir à ces étudiants une éducation identique à celle reçue par les blancs, autrement dit une éducation de type 
liberal arts. Les leaders de la communauté noire estiment que les futurs ministres du culte, avocats, médecins, dirigeants politiques et hommes d'affaires du pays doivent acquérir, au même titre que leurs pairs blancs, les traditions académiques et refusent l'idée d'une formation industrielle et agricole rudimentaire comme alternative réaliste. Mais du fait de la quasi absence d'écoles élémentaires et secondaires publiques pour les noirs dans le Sud, le nombre d'étudiants reste minuscule. Seules les universités Howard et Fisk proposent dans les faits la formation classique de type liberal arts de niveau universitaire. Face à une telle situation, de nombreux philanthropes se mettent à prôner une éducation technique mieux adaptée aux réalités des possibilités économiques et sociales (très limitées) dont disposent les noirs du Sud. Le modèle à cet égard est l'Institut Tuskegee fondé par l'éducateur Booker T. Washington en Alabama en 1881. La philosophie de cet établissement très populaire de formation essentiellement agricole inspire la création des land-grant colleges pour étudiants noirs qui se multiplient dans le Sud après la décision de la Cour Suprême américaine Plessy contre Ferguson de 1896 officialisant la constitutionnalité de la ségrégation en affirmant le principe du "separate but equal »: dès lors, pour ne pas avoir à accepter des noirs dans les écoles et les universités publiques de leur Etat, les gouvernements du Sud s'empressent de créer des colleges séparés mais dotés, sur le papier, de ressources de nature identique et de fonds distribués sur une base « juste et équitable». Mais pour les noirs, la réalité de la situation est bien celle de la séparation, pas celle de l'égalité de traitement et la démocratisation de l'enseignement supérieur reste largement une chimère jusqu'à la Seconde Guerre mondiale.

Malgré ces limites importantes, la démocratisation est lancée et elle ne s'arrête pas. Parallèlement à ces avancées, l'enseignement supérieur se restructure à la fin du XIXe siècle sous l'effet de deux facteurs majeurs. Premièrement, l'industrialisation et le rapide développement économique du pays donnent aux nouveaux savoirs techniques et scientifiques une importance accrue dans la société. Il est de plus en plus admis que l'université doit se moderniser pour répondre aux besoins du monde économique. Deuxièmement, c'est aussi l'époque de l'arrivée aux Etats-Unis du modèle universitaire allemand reconnu comme le meilleur d'Europe et mettant l'accent sur la recherche scientifique. Cette popularité se traduit dans l'enseignement supérieur américain par la création d'une nouvelle institution: "l'université » de recherche, dans laquelle les enseignants sont avant tout des chercheurs dont l'indépendance doit être préservée, et qui développe un enseignement de troisième cycle, par opposition au simple "college " centré sur l'enseignement et dispensant une formation de type undergraduate sans véritable activité de recherche. Ce nouveau type d'établissement séduit en particulier les nouveaux établissements urbains, telle Johns Hopkins University qui, entièrement créée sur ce modèle, propose uniquement des enseignements de niveau graduate. C'est donc depuis la fin du XIXe siècle et le début du XXe siècle qu'il existe aux Etats-Unis de grandes universités de recherche et un maillage étroit d'établissements spécialisés dans l'enseignement undergraduate de type liberal arts ou semi-professionnel. Cette division du paysage de l'enseignement supérieur américain se poursuit jusqu'à aujourd'hui.

\section{L'impact du G.I. Bill en 1945 : massification et évaluation}

17 Si la démocratisation de l'enseignement supérieur continue dans la première moitié du XXe siècle avec notamment l'accès croissant des étudiants juifs (dont le nombre devient significatif dans les années 1910 malgré les tests et les quotas spécifiques) et des 
femmes (dont la proportion dans le nombre total d'undergraduates dépasse $47 \%$ en 1920), le tournant clé est la loi dite du G.I. Bill de 1944 garantissant aux anciens combattants une année d'enseignement supérieur gratuite par période de 3 mois passée dans l'armée, à concurrence de 48 mois. Dans le cadre de cette loi, les frais d'inscription et autres frais, ainsi que l'achat de livres et de fournitures pour un montant total maximal de 500 dollars par an sont payés directement au college ou à l'université (à une époque où les frais d'inscription des institutions privées s'élèvent à environ 300 dollars et ceux des universités d'Etat sont considérablement moins élevés). L'étudiant reçoit aussi une indemnité de subsistance. Le succès de la loi est immédiat: dès 1946, les inscriptions à l'université dans ce cadre dépassent 1 million et les versements du gouvernement fédéral s'élèvent à plus de 5,5 milliards de dollars. En 1950, sur les 14 millions d'anciens combattants éligibles, plus de 2 millions ont choisi de poursuivre des études supérieures.

Le GI Bill constitue une innovation majeure dans l'histoire de l'enseignement supérieur américain à plusieurs titres: tout d'abord, cette loi fait de la poursuite d'études supérieures un droit, et non plus ce qu'elle était jusqu'alors, un privilège. En d'autres termes, il n'y a pas de limite au nombre de bénéficiaires. De plus, ce droit, étant accordé à l'étudiant, est transférable avec lui : un vétéran peut donc choisir de postuler pour s'inscrire à Harvard, mais aussi dans un community college proche de son domicile, dans une faculté de droit ou de médecine, ou encore dans une école d'ingénieur. Pour éviter tout abus du système, l'établissement doit être reconnu par le gouvernement fédéral, ce qui entraîne, conséquence indirecte du GI Bill, la mise en place d'un système d'évaluation des universités. Soucieuses de maintenir leur indépendance face au gouvernement fédéral, et alors que conformément au $10^{\mathrm{e}}$ amendement à la Constitution américaine qui déclare que « les pouvoirs qui ne sont pas délégués aux États-Unis par la Constitution, ni refusés par elle aux États, sont conservés par les États respectivement ou par le peuple", les universités choisissent de se soumettre volontairement à des instances autonomes d'évaluation. C'est le début du système d'accréditation régional et de son cycle d'évaluation institutionnelle tous les dix ans qui devient progressivement la procédure standard pour tous les établissements décernant des diplômes, en particulier s'ils souhaitent être éligibles à des financements fédéraux. Autre changement induit par le GI Bill la manière dont les universités et colleges évaluent les candidatures d'étudiants prospectifs : devant le nouvel afflux de demandes, la décision d'admission doit désormais être prise rapidement. Or, celle-ci est d'autant plus difficile à prendre que les anciens combattants ne disposent souvent pas des relevés de notes des étudiants classiques. Se met alors en place un système d'admissions recourant de plus en plus à des tests standardisés et prenant en compte aussi l'expérience autre que celle acquise au lycée.

19 Autres conséquences très importantes du GI Bill : l'expansion physique des universités qui doivent à la hâte construire de nouveaux bâtiments pour les cours et le logement, des laboratoires, des installations sportives, mais aussi adapter leurs locaux aux besoins spécifiques des étudiants vétérans parfois handicapés; tout aussi saisissante pour l'observateur de l'époque est la transformation de la culture étudiante sous l'effet de l'arrivée massive de cette nouvelle cohorte : plus âgés que l'étudiant traditionnel de 17 à 21 ans, les étudiants arrivés à l'université par le GI Bill ont aussi un rapport plus pragmatique aux études et les universités sont promptes à diversifier leur offre de cours pour répondre à leur désir d'acquérir une formation leur permettant d'obtenir 
immédiatement un bon emploi dans l'économie en pleine expansion de l'après-guerre. Leur arrivée signifie aussi la masculinisation du campus (même si quelque 60000 anciennes combattantes en bénéficient également) après les avancées des décennies précédentes : il faut attendre les années 1970 pour que les femmes représentent de nouveau $40 \%$ des étudiants undergraduate du pays. Cette proportion s'élève à $55 \%$ aujourd'hui.

20 L'impact considérable du GI Bill ne doit toutefois pas être exagéré car il s'accompagne d'une limite cruciale: son influence faible sur les relations raciales dans les colleges et les universités. Certes les GI noirs étaient éligibles et le nombre d'étudiants afroaméricains augmenta nettement grâce à ce dispositif, mais les termes de la loi ne comprenaient aucune obligation de non-discrimination pour les institutions participantes. Les établissements ayant traditionnellement exclu les minorités raciales ont donc continué de le faire en toute impunité (le gouvernement fédéral pratiquant lui-même jusqu'en 1948 la ségrégation dans ses forces armées). Malgré les avancées démocratiques du GI Bill, la doctrine "séparé mais égal» continue de guider les relations raciales à l'université comme dans les autres institutions américaines. Cette situation perdure jusqu'à l'adoption par le Congrès de la grande loi sur les droits civiques de 1964 qui interdit la ségrégation et la discrimination encore pratiquées en dépit de la décision de la Cour Suprême Brown contre le bureau de l'Education de Topeka, Kansas de 1954.

21 Cela dit, le GI Bill doit être vu comme la première étape d'un rôle nouveau pour le gouvernement fédéral dans l'enseignement supérieur qui se poursuit jusqu'à aujourd'hui. Dès 1947, le président Harry Truman commande une étude publiée sous le titre Higher Education for American Democracy qui souligne l'importance nationale de l'enseignement supérieur dans le nouveau contexte de la Guerre froide, aussi bien en matière sociale que pour l'influence des Etats-Unis dans le monde et pour la défense du pays. Depuis 65 ans, cette vision ne s'est guère modifiée; elle s'est même renforcée, forte de la croissance spectaculaire des universités américaines depuis la Seconde Guerre mondiale.

Après le deuxième conflit mondial, l'enseignement supérieur américain bénéficie en effet de trente années glorieuses de prospérité, de prestige et de popularité. Le gouvernement fédéral continue sa politique de financement de la recherche scientifique dont l'importance est jugée stratégique pour le pays et qu'il a initiée avec le projet Manhattan de fabrication de la bombe atomique pendant la guerre. Il se met à financer des programmes de recherche et de formation en langues étrangères et sur différentes aires culturelles et passe des contrats de recherche avec un groupe important d'universités du pays chargées de mener pour son compte diverses missions de recherche appliquée. Il faut rappeler ici que cet argent fédéral ne remplace jamais les contributions financières des Etats fédérés qui restent l'instance gouvernementale principale chargée des affaires et du financement éducatifs à tous les niveaux. Toutefois, il n'en reste pas moins que dans les établissements bénéficiaires de ces contrats, la manne fédérale devient vite indispensable à leur fonctionnement, leur expansion et leur réputation, et que la vaste majorité des colleges et universités qui n'en bénéficie pas se retrouve alors dans l'obligation de trouver d'autres sources de financement et d'autres moyens de se distinguer de leurs concurrents pour attirer des étudiants en nombre toujours croissant mais de plus en plus désireux de choisir leur université en fonction de leurs intérêts et besoins propres. En plus d'offrir un niveau de 
financement inédit dans l'histoire de l'enseignement supérieur, cette nouvelle implication du gouvernement fédéral a aussi pour effet d'accroître la concurrence entre établissements et de forcer l'ensemble des établissements à développer une niche et une identité uniques.

Parallèlement à ce rôle de financement de la recherche pour des raisons d'intérêt national, le gouvernement fédéral reconnaît la double nécessité d'ouvrir l'enseignement supérieur à une proportion accrue de la population (inscrite dans la grande loi sur l'enseignement supérieur de 1965 qui crée notamment les bourses étudiantes) et de se doter d'établissements réputés dans le monde entier pour l'excellence de leur recherche et de leur formation. Le paysage universitaire déjà riche de l'avant-guerre se renforce alors avec la multiplication des community colleges, établissements de proximité dispensant une formation semi-professionnelle pour un coût très raisonnable, qui élargissent la base du système, tandis que le haut de la pyramide ne cesse de s'élever grâce à la capacité démontrée des colleges et universités de se doter de programmes avancés sélectifs à tous les niveaux, la sélection étant présentée comme gage de qualité. Parallèlement, c'est aussi la période où apparaît un nouveau type d'institution: les établissements privés à but lucratif de formation spécialisée ou professionnelle, et les dites corporate universities, créées à l'initiative de grandes entreprises.

Cet âge d'or, basé sur la démocratisation de l'enseignement supérieur et la puissance de l'université de recherche, est celui sur lequel se fonde encore aujourd'hui la réputation du modèle de l'enseignement supérieur américain. Un autre facteur est sans doute aussi la capacité du système à s'adapter au double défi de la massification causée par le baby-boom (on est passé de moins de 1,5 million d'étudiants avant la Deuxième Guerre mondiale à 7,9 millions en 1970) et des revendications des mouvements identitaires des années 1960 et 1970 (en particulier des minorités ethno-raciales et sexuelles) qui ont profondément transformé l'institution universitaire.

De fait, les structures de l'enseignement supérieur américain n'ont guère évolué depuis les années 1970, même si de nouveaux défis sont apparus avec la crise économique de 2008. Celle-ci a considérablement diminué les ressources disponibles pour l'enseignement supérieur (et l'éducation en général) dans les budgets de l'Etat fédéral et des Etats fédérés, tout en provoquant une nouvelle hausse des droits d'inscription mettant désormais l'enseignement supérieur hors de portée d'une proportion croissante de la population. Cette dangereuse remise en cause de décennies de démocratisation de l'accès à l'enseignement supérieur pose aujourd'hui une question jusqu'alors inimaginable: celle des limites du modèle américain et de sa pérennité. C'est dans ce contexte qu'il convient d'interpréter l'appel récent du président Obama à relancer le développement de l'enseignement supérieur américain en misant non plus sur l'excellence des établissements d'élite comme moteur du système, mais sur les community colleges. ${ }^{10}$

Dans un pays où la population étudiante s'élève en 2010 à 19,1 millions de personnes (contre 13,8 millions en 1990, ce qui représente aujourd'hui plus de 41\% des 18-24 ans) réparties dans 4352 établissements et où le coût des études a plus que doublé depuis 1990 (en 2010, le coût moyen annuel des frais d'inscription et de logement dans les colleges et universités publics pour les étudiants de l'Etat est d'un peu moins de 15000 dollars contre un peu plus de 40000 dans les établissements privés), on comprend 
l'intérêt de cette réorientation des priorités nationales. Une leçon sans doute à méditer ailleurs également.

Paradoxalement, la mise en place des systèmes standardisés d'évaluation date des années 1945 avec le GI Bill, qui fut un levier pour la démocratisation du système : la rationalisation du système s'est imposée pour faire face à la croissance numérique du système et pour contrôler la circulation des fonds publics. Au fond, le système d'éducation supérieur nord-américain fonctionne d'emblée à plusieurs vitesses et repose sur la multiplication d'institutions spécialisées (que ce soit au niveau des universités ou des départements) pour intégrer différents secteurs de la population. Les lecteurs du dossier pourront ainsi comprendre le développement des départements de «Black Studies » aux Etats-Unis dans les années soixante grâce à l'article de Caroline Rolland, tandis que Ramón Grosfoguel analyse les dilemmes actuels des départements d'ethnic studies. Des dilemmes que les universités indigènes du Mexique (articles de David Lehmann) et de Bolivie (article d'Anders Burman) partagent en grande partie. Mais en partant d'une histoire universitaire différente, dont les racines plongent dans l'Espagne médiévale.

\section{Le système d'enseignement universitaire latino- américain à la croisée des modèles européens et états-uniens}

Quelques grands repères historiques ${ }^{11}$ peuvent être ici donnés à titre de comparaison avec le processus nord-américain. Avant 1492, des institutions spécialisées dans la formation savante des élites sont repérables dans les sources concernant les empires inca et aztèque. Avec la conquête espagnole, se diffuse en s'adaptant le modèle de l'université médiévale, scholastique, incarné en Espagne par Salamanque (Studium Generale, 1218) et Alcala de Henares (Estudio de escuelas generales, 1293). Au départ, les cours sont dispensés dans le cloître de la cathédrale et dans des maisons louées de la ville. Au début du XVe siècle sont construits des colegios (bâtiments qui incluent un internat pour les étudiants pauvres et boursiers mais aussi des salles de cours et des espaces de vie sociale, culturelle et sportive). Les colegios menores préparent au bachiller et les colegios mayores donnent des enseignements de licence et doctorat mais les examens se passent à l'Université. Au fil du temps, les colegios sont monopolisés par la noblesse. L'influence de Salamanque en particulier est perceptible à Lima (1575) et à Mexico (1595), à travers l'adoption de ses modes d'organisation, de ses programmes, de ses pratiques, de ses privilèges et de la circulation des enseignants. Lima et Mexico se livrent à une certaine compétition pour rayonner sur les autres universités régionales. La plupart des universités reçoivent des autorisations à la fois royales et pontificales. Elles sont souvent adossées à des séminaires, des couvents ou des collèges de dominicains, augustiniens ou jésuites ${ }^{12}$. Certaines universités sont dites mayores, oficiales et generales, avec des rentes de la real hacienda, avec de nombreuses chaires et privilèges ; les autres sont dites particulares, n'ayant pas la même faculté de délivrer des diplômes. Elles sont organisées en 4 facultades mayores (grandes facultés) : théologie, droit canon, droit, médecine, et une faculté mineure «Art et philosophie ». Un colegio tenu par des Franciscains, nommé Santa Cruz de Tlatelolco fut très tôt créé pour les élites indigènes de Mexico (1533) : pendant une cinquantaine d'années l'enseignement du latin mais aussi la mise au point d'un alphabet latin pour le nahuatl ont donné le 
jour à des œuvres scientifiques importantes basées sur les connaissances médicales et historiques nahua. Les premières générations d'indigènes formés dans le collège ont eu d'importantes responsabilités dans leur propre université et d'autres institutions coloniales avant que le modèle périclite. Ce modèle diffusionniste, qui insiste sur l'importation du modèle médiéval espagnol est par trop simpliste mais il rend compte néanmoins d'un fait important : la fondation rapide et durable de plus de vingt cinq universités sur tout le territoire de l'Amérique espagnole alors que dans le même temps la couronne portugaise n'a permis que l'ouverture de collèges et non d'universités, sans sur le sol brésilien jusqu'en 1808, date à laquelle la famille royale s'y installe

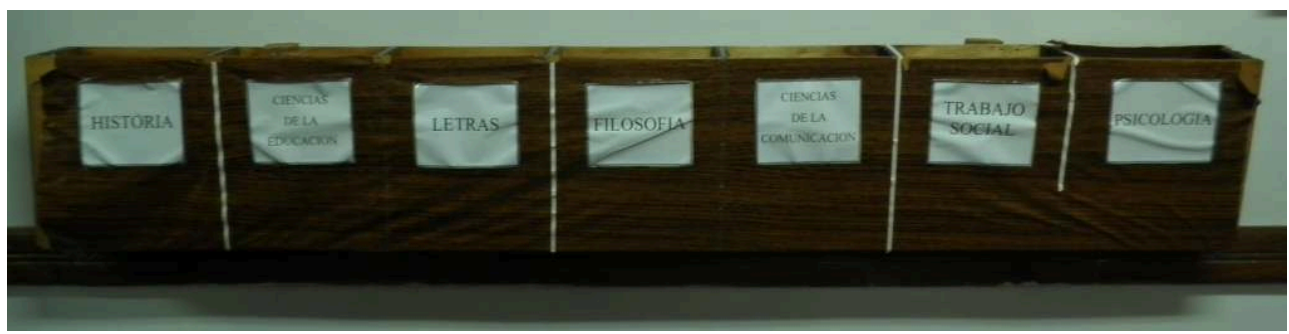

L'histoire de la construction des savoirs au concret : les boîtes aux lettres des sept départements de la faculté de Philosophie de l'Université Nationale d'Asunción.

Photo : C. Boidin, Paraguay 2011

Le vent de l'illustration française à la fin de la période coloniale souffle aussi fortement dans les universités latino-américaines avant que la période des indépendances républicaines ne diffuse les principes d'organisation napoléoniens (longues carrières professionnelles de 6 à 7 ans, sans diplômes intermédiaires) et le principe humboldtien de l'adossement de l'enseignement à la recherche. Le positivisme comtien a également eu un impact important, que ce soit du côté espagnol ou lusophone. Pour le $\mathrm{XX}^{\mathrm{e}}$ siècle, comme le rappelle l'article de Guadalupe Olivier dans ce dossier, trois moments sont importants : la réforme de Cordoba en 1918 défend le principe de la gratuité des études et la lutte pour la démocratisation d'une institution, pensée comme publique et autonome. La réforme des années 1970 entraîne la croissance d'institutions supérieures très différenciées qui ne sont pas nécessairement de type universitaire mais qui permettent d'ouvrir le système public. La troisième réforme s'accélère depuis les années 1990 avec la massification, la privatisation et l'internationalisation de l'éducation supérieure latino-américaine. 


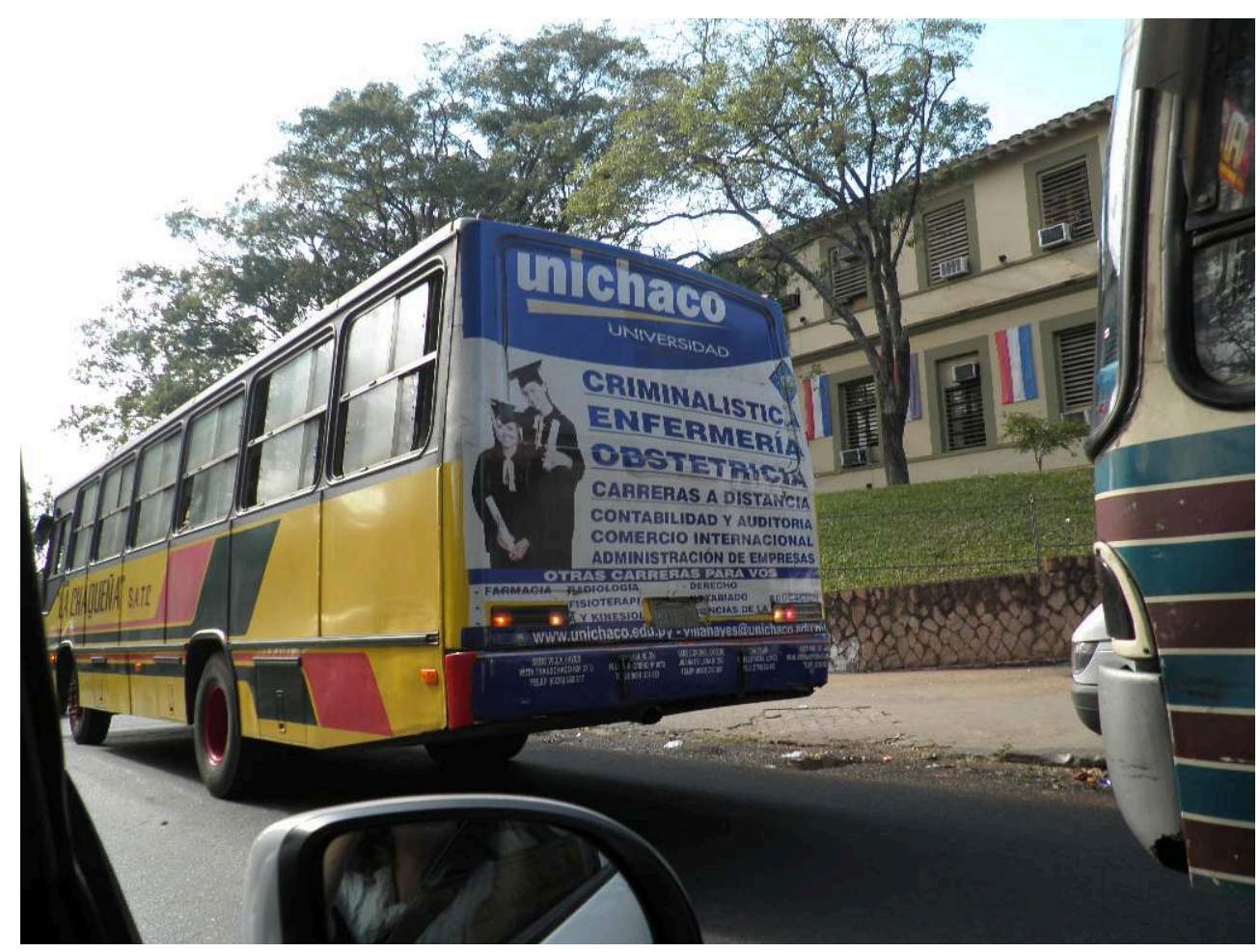

Multiplication des publicités pour les Universités privées en Amérique latine. Asunción, Paraguay, 2011.

Photo : C. Boidin 2011

\section{Massification, privatisation et développement des universités indigènes dans l'enseignement supérieur en Amérique latine}

Deux types d'analyses divergentes s'opposent: les unes, fondées sur une longue tradition de pensée critique en Amérique latine décryptent les politiques néolibérales contemporaines ${ }^{13}$ tandis que les autres multiplient les rapports synthétiques pour justifier leurs « recommandations", en matière de réformes (Banque Mondiale, Fond Monétaire international en particulier). Mais tout d'abord, quelques chiffres sur la massification et la privatisation accélérées du système de l'enseignement supérieur latino-américain ${ }^{14}$ :

31 Nombre d'universités et d'institutions d'enseignement supérieur (IES) en Amérique latine

195075 Universités

1975330 Universités

1985450 Universités

1995812 Universités (60,7\% privées) 5438 IES

20021213 Universités (69,2\% privées) 7514 IES

Aujourd'hui : probablement plus de 2000 Universités.

32 Nombre d'étudiants inscrits dans le système d'éducation supérieure en Amérique latine

1950267000

19701640000

19804930000 
19907350000

200011500000

200515293181

200917757000

Aujourd'hui (2012) environ 18 millions.

Le Brésil compte aujourd'hui 5,95 millions d'étudiants inscrits ${ }^{15}$, le Mexique et l'Argentine environ 2,5 millions chacun. Les trois pays totalisent environ 11 millions d'inscrits, soit $60 \%$ des 18 millions d'étudiants latino-américains aujourd'hui. Ce sont aussi les trois pays qui ont le nombre le plus conséquent d'étudiants en masters et doctorats. En nombre total d'étudiants, suivent la Colombie (1,3 million), le Venezuela (1 million), le Pérou (900 000), le Chili (670 000), Cuba (470 000), la Bolivie (350 000). Le taux d'inscription dans l'enseignement supérieur latino-américain s'est considérablement amélioré, passant de 17\% au début des années 1990 à 34\% aujourd'hui. La situation varie considérablement d'un pays à l'autre: de $18 \%$ au Guatemala, 27\% au Mexique, 30\% au Brésil, 32\% en Colombie, 35\% au Pérou, 52\% au Venezuela, 67\% en Argentine jusque 88\% à Cuba. Autrement dit, alors que le Mexique et l'Argentine ont le même nombre d'étudiants, le Mexique est loin de couvrir autant que l'Argentine les expectatives de sa population. Par ailleurs certains systèmes d'enseignement sont particulièrement inégalitaires : les familles de faibles ressources économiques sont exclues des universités publiques de qualité, faute d'avoir pu inscrire leurs enfants dans de bonnes écoles secondaires (privées ou publiques). C'est particulièrement le cas au Brésil mais aussi au Mexique, au Chili et en Equateur. L'investissement annuel global par étudiant latino-américain est aujourd'hui de 2380 dollars. Autrement dit, si en nombre total d'étudiants l'Amérique latine et les EtatsUnis pèsent singulièrement le même poids (18 millions contre 19 millions), les premiers sont considérablement moins financés (Pour mémoire les frais d'inscription aux Etats Unis varient de 15000 à 40000 dollars annuels).

Cette massification que certains peuvent qualifier de démocratisation n'aurait pas pu avoir lieu sans la multiplication d'universités privées, qu'elles soient sans but lucratif ou clairement orientées vers la captation des investissements éducatifs que de plus en plus de familles latino-américaines sont disposées à réaliser pour leurs enfants. Les processus d'intégration dans les Amériques entraînent avec eux la création d'associations entre institutions d'éducation supérieure de plusieurs pays, publiques ou privées (Article de Guadalupe Olivier dans ce dossier) ${ }^{16}$ et la multiplication de rapports comparatifs. 


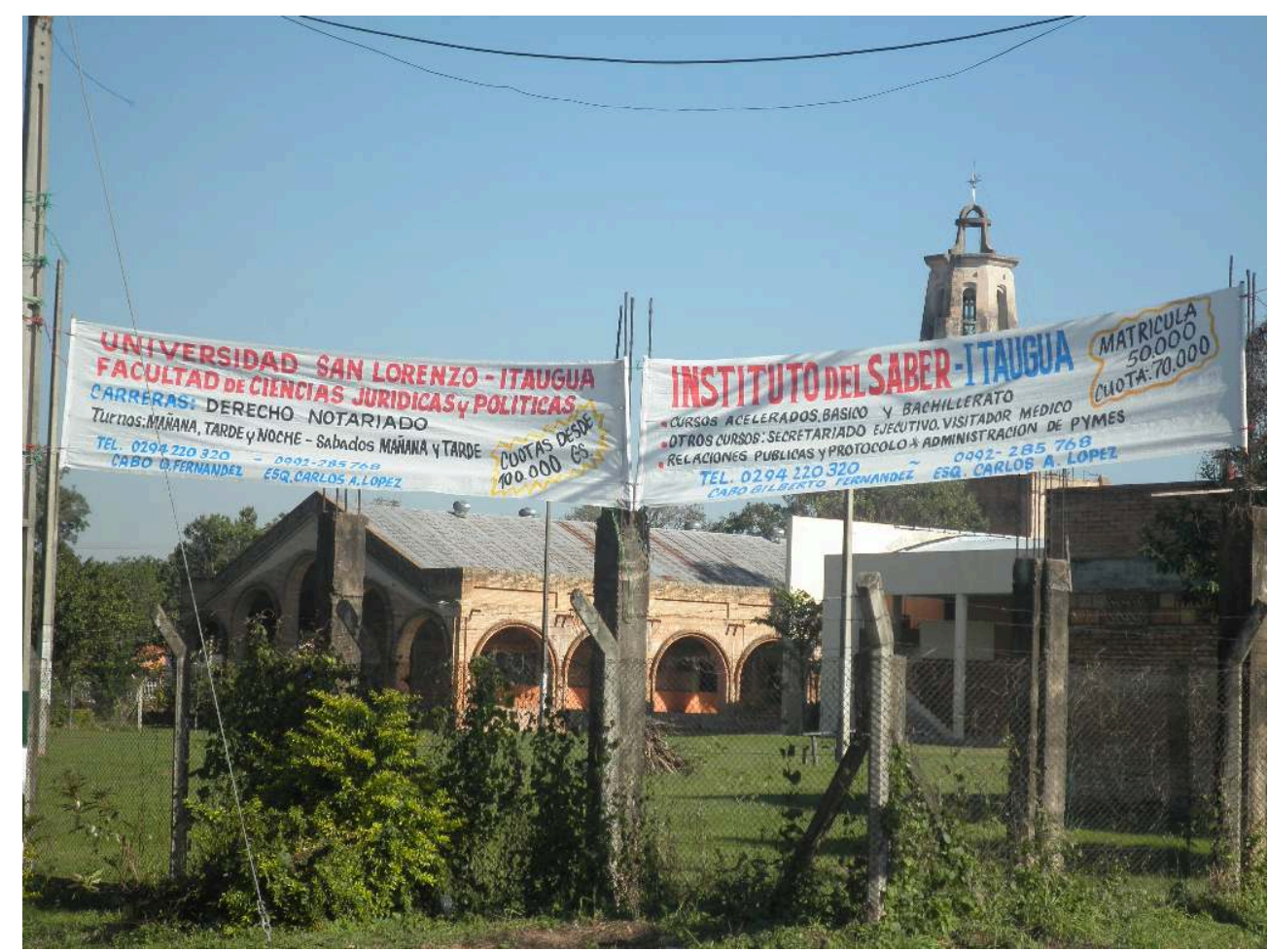

Publicité pour une Université d'un côté et un « Institut du savoir » de l'autre, San Lorenzo, Paraguay. Photo : C. Boidin 2011

Depuis les années 1960, différents organismes de l'UNESCO mais aussi la CEPAL (Comisión Económica para América Latina y el Caribe) ont impulsé des études comparatives des systèmes d'enseignement à l'échelle latino-américaine. A partir de 1978, Le Centro Regional para la Educación Superior en América Latina y el Caribe (CRESALC), transformé à la fin des années 1990 en Instituto Internacional para la Educación Superior en América Latina y el Caribe (IESALC), a produit de nombreux rapports comme, en 1995, El Documento de Política para el Cambio y el Desarrollo de la Educación Superior qui a conduit l'UNESCO en 1998 à définir un plan d'action organisé autour de cinq axes: 1) la pertinence sociale des enseignements, 2) la qualité, l'évaluation, 3) la gestion et le financement, 4) l'utilisation des nouveaux moyens de communication; et 5) la coopération internationale. Ces cinq axes font singulièrement penser à ceux que l'Union européenne s'était fixée en 1999, à la différence près que la dimension sociale des enseignements est proposée comme prioritaire, avant la volonté d'évaluation.

Finalement, quelques années avant l'Europe, plusieurs pays d'Amérique latine ont largement libéralisé leur système d'enseignement supérieur, mis en place les systèmes standardisés d'évaluation de la recherche et de l'enseignement, tout en multipliant les institutions principalement destinées aux populations autochtones: les universités dites «indigènes » ou «interculturelles » suivant les pays et les périodes, à propos desquelles réfléchissent autant Anders Burman pour la Bolivie et David Lehmann pour le Mexique dans ce dossier. 


\section{Déprovincialiser les universités européennes}

37 En France, la réforme des universités accélérée en 2007 avait alors soulevé une levée de boucliers et réuni sur le pavé des manifestants de tous bords politiques, droite/gauche. Le désir de défendre une certaine idée du savoir scientifique et de l'enseignement supérieur en tant que «biens collectifs et publics » et non pas en tant que «bien de consommation individuel» (un diplôme valable et utile dans la carrière de l'individu) réunissait aussi bien les sciences humaines et sociales que les sciences juridiques, les sciences physiques, mathématiques et naturelles. Passer d'une relation pédagogique à une relation marchande où l'étudiant devient un client va à l'encontre d'une culture universitaire européenne. Mais dans l'opinion publique cette forme de défense a pu être perçue comme un refus de la part des universitaires de préparer leurs étudiants à une intégration dans le monde professionnel. Et pourtant, il ne s'agissait pas de réagir contre l'intégration professionnelle des étudiants : une formation rigoureuse aiguise le sens critique et l'autonomie des étudiants, les préparant par là même à s'adapter intelligemment à toute situation sociale et en particulier professionnelle. Il s'agissait alors de réagir contre l'importation d'une culture entrepreneuriale au sein de l'université et la transformation de l'université en entreprise.

38 Au final, cette série de malentendus et de confusions nous a conduits à nous demander si la crise des universités françaises, mais plus largement européennes, n'avait pas sa source au-delà des pressions engendrées par le processus de Bologne. Elle viendrait également d'un essoufflement du modèle universitaire et de l'universalisme nés des Lumières. De fait, les critiques internes envers les savoirs et le rapport aux savoirs pratiqués dans les universités ne sont pas nouvelles (Foucault, Bourdieu, Lyotard, Latour, Rancière et d'autres...). Toutefois, depuis une dizaine d'années, le modèle de l'Université kantienne-humboldtienne (" la science par et pour la science », détachée de la théologie ; caractère encyclopédique des recherches ; figures du chercheur enseignant et de l'étudiant-chercheur) est aussi largement interrogé et critiqué par les penseurs postcoloniaux asiatiques, latino-américains, nord-américains et européens. En particulier, les auteurs latino-américains, qui préfèrent se dire décoloniaux que postcoloniaux, interrogent l'eurocentrisme épistémique qui guident certaines pratiques de l'institution. Ils dénoncent l'eurocentrisme, le racisme et le sexisme épistémiques d'institutions ou seules les idées élaborées à partir des traditions occidentales et publiées par des hommes européens ou euro-américains sont considérées comme étant capables d'universalité. Plus profondément, ils questionnent la volonté de savoir encyclopédique totale, en particulier anthropologique, qui si elle relève d'une démarche de connaissance des " autres " n'aboutit pas à leur pleine reconnaissance comme sujets et auteurs. Or cette critique ne conduit pas nécessairement à un relativisme étroit et à l'abandon de toute recherche universelle. $\mathrm{Au}$ contraire, l'intérêt des dernières réflexions des penseurs latino-américains postcoloniaux est qu'ils soulignent la nécessité d'un processus de réflexion universelle qui se construise par le dialogue entre chercheur(e)s issus d'horizons épistémiques pluriels.

39 Les réformes proposées par le processus de Bologne n'affrontent précisément pas ces critiques profondes. Au contraire, en se focalisant essentiellement sur les méthodes de gestion et d'évaluation des enseignants et des diplômes, elles renforcent le désenchantement du monde universitaire. Pourtant le potentiel de renouvellement des 
universités françaises et européennes est considérable. En particulier en s'ouvrant plus résolument dans des dialogues inter-épistémiques où l'enjeu serait de construire une nouvelle écologie des savoirs. Loin de se cantonner à un relativisme mou et par défaut, ou à des "petits récits", l'objectif serait de rechercher des savoirs universels, mais en passant par la mise en place de dialogues horizontaux entre différentes traditions de pensées. Réformer l'université en recherchant un universalisme moins provincial et plus ouvert implique une refonte profonde de nos manières de penser et de nos découpages disciplinaires. La création du campus Condorcet pourrait être l'occasion de chercher des formes de pensée pluri-verselles et de rester en prise avec les questions posées par la société ${ }^{17}$. Ouvertures et nouvelles constructions des savoirs qui seraient rendues visibles par l'architecture choisie pour ce Campus. Les conversations avec Cynthia Ghorra-Gobin et João Sette Whiteker Ferreira concernant l'organisation des campus californiens et brésiliens respectivement permettront de saisir par contraste les spécificités d'un campus localisé dans le nord du grand Paris et ouvert sur la vie de quartiers populaires en pleine reconstruction comme Aubervilliers et Saint Denis.

C'est la raison pour laquelle ce dossier (et la conversation campus qui l'accompagne) se propose d'entamer le dialogue avec d'autres traditions de pensée, en particulier latinoaméricaines et d'autres expériences comme les universités indigènes latinoaméricaines. En effet, si les "corporate universities " des États-Unis sont érigées en modèle à partir des années 1970, l'Amérique latine va très rapidement s'en saisir et les multiplier dans les années 1980, tout en ouvrant la possibilité à des universités autonomes et indigènes de se constituer. Autrement dit, l'analyse et la discussion des transformations universitaires que connaissent d'une part le continent américain depuis quatre décennies et l'Europe depuis une dizaine d'années devraient nous permettre de mieux comprendre la situation que nous traversons et de mieux penser l'université européenne de demain.

\section{NOTES}

1. Nous jouons ici avec le sens que Dipesh Chakrabarty donne à l'expression Provincialiser l'Europe - La pensée postcoloniale et la différence historique, Paris, Editions Amsterdam, 2009, traduction de Olivier Ruchet et, Nicolas Vieillescazes. Pour Dipesh Chakrabarty qui pense la situation indienne “La pensée européenne est aussi indispensable qu'inadéquate pour penser l'expérience de la modernité politique dans les nations non occidentales, et provincialiser l'Europe devient une tâche consistant à examiner la manière dont il est possible de renouveler cette pensée, qui constitue à présent notre héritage à tous et qui nous affecte tous, à partir des marges et pour elles. Mais les marges sont bien sûr tout aussi plurielles et diverses que les centres." (p. 53) Pour nous qui pensons depuis l'Europe, déprovincialiser les Universités Européennes consiste à reconnaître, humblement, la nécessité vitale de se "brancher", de se laisser renouveler par les traditions de pensée les plus diverses. Nous l'explicitons d'avantage à la fin de cet édito.

2. Pour un classement récent des universités latino-américaines entre elles: http://americalatina.blog.lemonde.fr/2011/10/05/l'universite-de-sao-paulo-en-tete-du-premier-classementlatino-americain/ 
3. http://www.cpihts.com/PDF/Declaracao\%20de\%20la\%20Sorbonne.pdf consulté le 2 juin 2012.

4. http://eacea.ec.europa.eu/education/eurydice/documents/thematic_reports/122FR.pdf

5. Isabelle Bruno, "Y a-t-il un pilote dans l'Union ? Tableaux de bord, indicateurs, cibles chiffrées : les balises de la décision ", Politix, vol. 82, n², 2008, p. 95-118.

6. Communiqué de Londres, 2007, p. 5 cité dans le rapport de la Commission européenne, La modernisation de l'enseignement supérieur en Europe : financement et dimension sociale, 2011, p. 9.

7. (Généralisation des cursus en trois phases Licence, Master, Doctorat et de la capitalisation d'ECTS tout au long de son parcours quelle que soit l'université et la région).

8. Pas entièrement nouvelle puisque la Free University of New York City est fondée en 1847 par exemple, la tendance s'accélère après la Guerre de Sécession avec la naissance des universités de Cincinnati, de Hunter College à New York, de Wayne University à Détroit, de Boston University, Temple University à Philadelphie, ou encore George Washington University à Washington.

9. A cet égard, les nouvelles universités publiques jouent un rôle pionnier, avec tout d'abord l'université d'Iowa (1855), de Wisconsin (1863), suivie par les universités de l'Indiana, du Missouri, du Michigan et de Californie dans les années 1870. Dès cette décennie, la plupart des établissements d'enseignement supérieur de l'Ouest et du Midwest acceptent cette pratique et les résistances se concentrent surtout dans les universités de l'Ivy League du Nord-Est.

10. Comme le dit bien Dominique Godrèche « accessibles à tous, sans limite d'âge ni distinction sociale ou de provenance académique, les community colleges représentent aux Etats-Unis le remède à un enseignement universitaire aussi cher que sélectif. Leur cursus sur deux ans prépare, au sortir du lycée, à l'associate's degree nécessaire pour obtenir - après deux années à l'université - le bachelor's degree (équivalent de la licence). Il offre ainsi aux élèves issus des classes défavorisées, qui ne pourraient financer quatre ans d'études dans une université privée, un accès à l'enseignement supérieur. De plus, ces colleges dispensent des enseignements professionnels sanctionnés par des certificates, ainsi qu'une éducation diversifiée, à une frange de la population qui autrement n'aurait droit à rien, notamment les immigrés. [...] L'intégration des community colleges dans le tissu économique de leur région et la réactivité dont ils font preuve pour adapter les cours aux besoins nouveaux représentent pour eux des atouts d'importance ». Voir Dominique Godrèche, «En marge des universités, les community colleges » et « Onze millions d'étudiants », Le Monde diplomatique, juin 2010.

11. Pour une première approche : Celina Lértora Mendoza, «Lineamientos para una historia de la universidad latinoamericana", Cuadernos de la Facultad de Humanidades y Ciencias Sociales, diciembre, $\mathrm{n}^{\circ} 15, \mathrm{pp} .43-51$. Pour aller plus loin : Diana Soto Arango, Manuel Lucena Salmoral y Carlos Rincón (dir.), Estudios sobre la universidad latinoamericana. De la colonia al siglo XXI, RUDECOLOMBIA, HISULA, Universidad Pedagógica y tecnológica de Colombia, Ed. Doce Calles, 2003. 12. Voir Aguedas Rodriguez Cruz, la proyeccion de la universidad de salamanca en Hispanoamérica, sans date, postérieur à 2000, consulté sur http://www.americanistas.es/biblo/textos/s04/ s-04-10.pdf ; La Universidad en la América hispánica, Madrid, Mapfre, 1992.

13. Voir en particulier les travaux de Mollis, M. (comp.), Las universidades en América latina: reformadas o alteradas?, Buenos Aires, CLACSO, 2003.

14. Francisco Lopez Segrera, «La educacion superior en el mundo y en América latina y el Caribe », Revista paraguaya de sociologia, année 47, n¹36, janvier-juin 2010. Lopez Segrera est une des principales références pour ses rapports UNESCO sur l'éducation supérieure en AL depuis les années 2000. Lorsque les chiffres qui suivent ne viennent pas de son article nous le précisons.

15. http://gestao2010.mec.gov.br/indicadores/chart_60.php données élaborées par le Ministère de l'enseignement supérieur brésilien.

16. http://america-latina.blog.lemonde.fr/2011/10/05/l'universite-de-sao-paulo-entete-du-premier-classement-latino-americain/ 
17. Ces principes sont proclamés comme suit dans le programme scientifique du Campus: «Réunir les humanités et les sciences sociales ; Conjoindre étude du passé et étude du présent ; Promouvoir des questionnements transversaux, adaptés à l'analyse des dynamiques d'un monde global. Ces principes sont associés à deux orientations : vers les objets (aires culturelles, religions, textes, territoires, populations, etc.) préférés aux disciplines et vers la création d'un outil d'expertise et d'intelligence du contemporain, associé à un élargissement et à un renouvellement des débouchés dans le domaine des sciences humaines et sociales ». http://www.campuscondorcet.fr/campus-condorcet/La-politique-scientifique/Le-programme-scientifique/p-451-Leprogramme-scientifique.htm

\section{INDEX}

Mots-clés : Universités, Amérique du Nord, Brésil, Enseignement supérieur, colonialité du savoir, Amérique latine

Palabras claves : Universidad, América Latina, América del norte, Brasil, Educación Superior Keywords : University, Latin America, North America, Brazil, Higher Education, coloniality of knowledge

\section{AUTEURS}

\section{CAROLINE ROLLAND-DIAMOND}

Maître de conférences en histoire à l'Université Paris Ouest Nanterre. Ses axes de recherche portent sur l'histoire sociale, l'histoire politique et l'histoire urbaine des États-Unis au XXe siècle, ainsi que sur l'histoire des mouvements étudiants américains depuis les années 1960, l'histoire de l'institution universitaire, la théorie des mouvements sociaux et l'histoire de la répression politique. Elle est l'auteur de Chicago : le moment 68. Territoires de la contestation étudiante et répression politique (Paris: Syllepse, 2011) et a co-dirigé (avec Robi Morder) Etudiant(e)s du monde en mouvement. Migrations. Cosmopolitisme et internationales étudiantes (Paris, Syllepse, 2012).

rolland.carolineatgmailpointcom

\section{CAPUCINE BOIDIN}

Maître de conférences en anthropologie à l'Université Sorbonne Nouvelle Paris 3, IHEAL (Institut des Hautes Etudes d'Amérique Latine). Chargée de cours de guarani à l'INALCO (Institut National des Langues et Civilisations Orientales), ses axes de recherche portent sur le métissage, le genre, les mémoires de guerre. Elle a publié en 2010 un numéro des Cahiers des Amériques latines sur le tournant décolonial.

http://www.ladocumentationfrancaise.fr/catalogue/3303332400621/index.shtml ;http:// www.iheal.univ-paris3.fr/spip.php?article1041

\section{JAMES COHEN}

Professeur à l'Institut du monde anglophone et enseignant à l'Institut des hautes études de l'Amérique Latine, Université de Paris 3 Sorbonne Nouvelle. Spécialiste des questions de race/ 
ethnicité et d'immigration aux Etats-Unis et du rôle des Etats-Unis dans les Amériques.

jim.cohenatlibertysurfpointfr

\section{RAMON GROSFOGUEL}

Maître de conférences (Associate Professor) en études ethniques à l'université de Californie, Berkeley et chercheur invité auprès de la Maison des sciences de l'homme à Paris. Il a publié de nombreux articles et ouvrages sur l'économie politique du système mondial et sur les migrations caribéennes en Europe occidentale et aux États-Unis. Grosfogu (at) Berkeley point edu. 